\title{
Solution of the Fokker-Planck Transport Equation by Matrix Factorization*
}

\author{
A. Andrade and T. A. Oliphant \\ Los Alamos Scientific Laboratory, Los Alamos, New Mexico 87544 \\ AND \\ T. KAMMASH \\ Department of Nuclear Engineering, University of Michigan, \\ Ann Arbor, Michigan 48109 \\ Received January 5, 1981
}

\begin{abstract}
A matrix factorization method is used to solve the Fokker-Planck (Landau) charged particle transport equation. By treating all phase space variables as discrete in analogy to $S_{n}$ neutronics, the collision term takes on a five-point difference form which is readily treatable by this method. In order to illustrate this technique, the energy deposited by fast ions in a geonetrically spherical, Maxwellian background plasma is calculated. Although this technique can be generalized to other geometries, its essential elements are best iliustrated in this simple context.
\end{abstract}

\section{INTRODUCTION}

In the study of charged particle transport in plasmas, numerical techniques for solving the Fokker-Planck equation have been developed which closely parallel those used in neutron transport. This was a natural step in the development of solution methods in charged particle transport (CPT) in view of the fact that the theory and methods of neutron transport have been well developed [1,2]. Moreover, since much of the pioneering work in CPT was carried out in conjunction with the ongoing effort to build controlled fusion devices, the early methodologies developed to solve the transport equation were made more applicable to those machines. In the well-known analysis of transport in mirror machines by Killeen and Marx [3], for example, the calculations of spatial changes along the magnetic field are based on an assumption that the distribution function of ions remain approximately constant along a guiding center orbit, an assumption which is sufficiently accurate and more appropriate for mirror confinement systems.

* Work supported by United States Department of Energy Contract W-7405-Eng. 36. 'lhe U.S. Government's right to retain a nonexclusive royalty-free license in and to the copyright covering this paper, for governmental purposes, is acknowledged. 
Other authors have used expansion methods $[4,5]$ or diffusion theory techniques [6] to solve the transport equation. The diffusion techniques require that sequential moments of the transport equation be taken so as to generate a coupled set of equations, and further require that a prescription for closing that set be given. The transport problem is then reduced to the solution of that set.

In yet other approaches, the techniques of neutron transport are used directly in attacking the CPT equation. This is done by either reformulating the Fokker-Planck collision term into a form which matches the structure of a standard neutronics code [7] such that existing computer programs can be used directly for CPT, or deriving cross sections [8], which simulate the slowing down of ions to be used in existing neutronics codes. In other methods $[9,10]$, the differencing and multigrouping techniques of neutronics are applied to yield solutions to the CPT equation by standard algorithms.

In this paper it is shown that the Fokker-Planck charged particle transport equation, when appropriately approximated by finite differences, can be solved in a fully implicit manner by a simple and efficient matrix factorization method. By treating the independent phase space variables as discrete in analogy to $S_{n}$ neutronics, the collision term takes a form which is readily treatable by this method. In order to illustrate this technique, the energy deposited by fast ions in a geometrically spherical, Maxwellian plasma is calculated. Although this technique is easily generalized to other geometries, its essential elements are best illustrated in this simple context.

In Section I of this article, the form of the transport equation to be solved is developed. In Section II, the difference approximation to this equation is derived and the matrix factorization solution technique is described in detail. In Section III the results of a calculation of the energy deposited by fast ions to a plasma are compared to those given in Ref. [7]. All quantities used herein have MKS units, while temperatures are expressed in $\mathrm{keV}$.

\section{The FokKer-Planck Transport Equation}

The equation that describes the evolution of a "test" distribution $f(\mathbf{r}, \mathbf{v}, t)$ of charged particles in the phase space of $\mathbf{r}$ and $\mathbf{v}$ during time $t$, due to small angle collisions with other particles of a background species $b$ distributed as $f_{b}(\mathbf{r}, \mathbf{v}, t)$, has come to be known as the Fokker-Planck equation and can be written as [11]

$$
\frac{\partial f(\mathbf{r}, \mathbf{v}, t)}{\partial t}+\nabla_{\mathbf{r}} \cdot \mathbf{v} f+\nabla_{\mathbf{v}} \cdot \mathbf{a} f=-\sum_{b} \nabla_{\mathbf{v}} \cdot \mathbf{J} .
$$

The acceleration a in the third term of this equation is the Lorentz acceleration due to externally imposed electromagnetic fields as well as the self-consistent fields created by the charged particles' collective actions or higher order collisional processes. In 
this analysis, these higher order effects will be ignored and problems with external fields will not be considered.

Although Eq. (1) is a nonlinear equation [11] for $f$, it can be reduced to a simple linear form by invoking the assumption that the "background" distributions $f_{b}$ remain isotropic for all time. It can then be written in spherical polar coordinates as

$$
\frac{\partial f(r, v, \mu, t)}{\partial t}+\frac{v \mu}{r^{2}} \frac{\partial}{\partial r}\left(r^{2} f\right)+\frac{v}{r} \frac{\partial}{\partial \mu}\left[\left(1-\mu^{2}\right) f\right]=\left(\frac{\partial f}{\partial t}\right)_{\mathrm{coll}},
$$

where

$$
\begin{aligned}
\left(\frac{\partial f}{\partial t}\right)_{\text {coll }} & =-\sum_{b}\left\{\frac{1}{v^{2}} \frac{\partial}{\partial v} v^{2} J^{v}+\frac{\partial}{\partial \mu} J^{\mu}\right\}, \\
-\frac{J}{4 \pi \Gamma Z_{b}^{2}} & =\frac{A}{A_{b}} \frac{f}{v^{2}} H_{b 2}(v)+\frac{1}{3} \frac{\partial f}{\partial v}\left\{\frac{H_{b 3}(v)}{v^{3}}+H_{b 1}(v)\right\}, \\
-\frac{J^{\mu}}{4 \pi \Gamma Z_{b}^{2}} & =\frac{1}{2 v^{3}}\left\{H_{b 2}(v)-\frac{H_{b 3}(v)}{3 v^{2}}+\frac{2 v}{3} H_{b 1}(v)\right\}\left(1-\mu^{2}\right) \frac{\partial f}{\partial \mu},
\end{aligned}
$$

and where $\mu$ is defined as $(\mathbf{v} \cdot \mathbf{r}) /|\mathbf{v}||\mathbf{r}|$. Note that the distribution function $f$ is allowed to have a one dimensional dependence in configuration space and two degrees of freedom in velocity space (azimuthal symmetry). Here $Z_{b}$ and $A_{b}$ are the atomic number and atomic weight of the background species and the factor $\Gamma$ is defined as in Rosenbluth et al. [12]. $H_{b 1}(v), H_{b 2}(v)$, and $H_{b 3}(v)$ are the standard integrals

$$
\begin{aligned}
& H_{b 1}(v)=\int_{v}^{\infty} u f_{b}(u) d u, \\
& H_{b 2}(v)=\int_{0}^{v} u^{2} f_{b}(u) d u, \\
& H_{b 3}(v)=\int_{0}^{v} u^{4} f_{b}(u) d u .
\end{aligned}
$$

For computational convenience, the scalings

$$
\begin{array}{ll}
\bar{n}=n / N_{0}, & \bar{v}=v / C_{0}, \\
\bar{r}=r / R_{0}, & \bar{t}=t / t_{0}
\end{array}
$$

are introduced for densities, velocities, radial distances, and time in this analysis so that the distribution functions will scale as $\bar{f}=f C_{0}^{3} / N_{0}$. In these new variables, the Maxwellian distribution has the form $f_{b}(u)=\left(n_{b} / \pi^{3 / 2} v_{0 b}^{3}\right) \exp \left(-u^{2} / v_{0 b}^{2}\right)$, where $v_{0 b}=$ $\left(T_{b} / A_{b}\right)^{1 / 2} \cdot N_{0}$, and $t_{0}$ are arbitrarily chosen to suit the problem at hand. $C_{0}$ is defined to be $\left[2 k T_{0} / m\right]^{1 / 2}$ and $R_{0}$ is chosen to equal $C_{0} \cdot t_{0}$, where $T_{0}$ is some arbitrary 
standard temperature, $k$ is Boltzmann's constant, and $m$ is the mass corresponding to one atomic mass unit.

By using Eqs. (7) to scale the transport equation, Eq. (2), it retains its form identically except for the factor $\Gamma$ which is replaced by $\bar{\Gamma}=\Gamma N_{0} t_{0} / C_{0}^{3}$, while the integrals, Eqs. (6), evaluted for a Maxwellian distribution, take on the functional forms

$$
\begin{aligned}
& H_{b 1}(v)=\frac{n_{b}}{2 \pi^{3 / 2} v_{0 b}} e^{-v^{2} / v_{0 b}^{2},} \\
& H_{b 2}(v)=\frac{n_{b}}{\pi^{3 / 2}}\left\{\frac{\sqrt{\pi}}{4} \operatorname{erf}\left(\frac{v}{v_{0 b}}\right)-\frac{v}{2 v_{0 b}} e^{-v^{2} / v_{0 b}^{2}}\right\}, \\
& I_{b 3}(v)=\frac{n_{b} v_{0 b}^{2}}{2 \pi^{3 / 2}}\left\{\frac{3 \sqrt{\pi}}{4} \operatorname{erf}\left(\frac{v}{v_{0 b}}\right)-\frac{v}{v_{0 b}}\left(\frac{3}{2}+\frac{v^{2}}{v_{0 b}^{2}}\right) e^{-v^{2} / v_{0 b}^{2}}\right\},
\end{aligned}
$$

where the barred notation for the scaled quantities has been dropped, here, and for the remainder of this analysis.

\section{The Difference Approximation to the TRANSPORT EQUATION AND ItS SOLUTION}

\section{A. The Difference Appromination}

A differencing operator [9] that treats each independent variable of phase space as discrete is

$$
\mathscr{R}=\frac{1}{\beta} \int_{t_{s}}^{t_{s} 11} d t \int_{r_{i-1 / 2}}^{r_{i 11 / 2}} r^{2} d r \int_{v_{g-1 / 2}}^{\eta_{\xi}+1 / 2} v^{2} d v \int_{\mu_{n-1 / 2}}^{u_{n+1 / 2}} d \mu,
$$

where $s, i, g$, and $n$ are the indices on times, zones, velocities, and angles respectively, and $\beta$ is defined to be the quantity $\Delta t_{s} \Delta r_{i}^{3} \Delta v_{g}^{3} \Delta \mu_{n}$, where $\Delta t_{s}=t_{s+1}-t_{s}, \Delta r_{i}^{3}=$ $\left(r_{i+1 / 2}^{3}-r_{i-1 / 2}^{3}\right) / 3, \Delta v_{g}^{3}=\left(v_{g+1 / 2}^{3}-v_{g-1 / 2}^{3}\right) / 3$, and $\Delta \mu_{n}=\mu_{n+1 / 2}-\mu_{n-1 / 2}$. The halfinteger quantities will be those at cell edges while integer quantities will be cell centered. Since the operator is of the implicit type, all quantities in the following analysis will be defined at the "updated" time $t_{s+1}$ and will be cell centered unless specified by a subsript to be otherwise.

Applying this operator to Eq. (2) yields the difference approximation

$$
\begin{aligned}
\frac{f-f_{s}}{\Delta t_{s}} & +\frac{\mu_{n}}{V_{i}} \frac{\Delta v_{g}^{4}}{\Delta v_{g}^{3}}\left[A_{i+1 / 2} f_{i+1 / 2}-A_{i-1 / 2} f_{i-1 / 2}\right] \\
& +\frac{1}{V_{i} \Delta \mu_{n}} \frac{\Delta v_{g}^{4}}{\Delta v_{g}^{3}}\left[\alpha_{n+1 / 2} f_{n+1 / 2}-\alpha_{n-1 / 2} f_{n-1 / 2}\right] \\
= & -\sum_{b}\left\{\frac{1}{\Delta v_{g}^{3}}\left[v_{g+1 / 2}^{2} J_{g+1 / 2}^{v}-v_{g-1 / 2}^{2} J_{g-1 / 2}^{v}\right]+\frac{1}{\Delta \mu_{n}}\left[J_{n+1 / 2}^{\mu}-J_{n-1 / 2}^{\mu}\right]\right\},
\end{aligned}
$$


where $V_{i}=\Delta r_{i}^{3}, A_{i+1 / 2}=r_{i+1 / 2}^{2}$, and where the alpha coefficients are defined by the recursion relation $\alpha_{n+1 / 2}=\alpha_{n-1 / 2}-\mu_{n} \Delta \mu_{n}\left[A_{i+1 / 2}-A_{i-1 / 2}\right]$ as in $S_{n}$ neutronics theory [1] in order to preserve the conservation of particles for finite intervals $\Delta \mu_{n}$.

Equation (10) can be written in a more concise form by defining the quantities $\xi=$ $\Delta v_{g}^{4} / \Delta v_{g}^{3} V_{i}, \quad A=\mu_{n} \Delta \mu_{n}\left[A_{i+1 / 2} f_{i+1 / 2}-A_{i-1 / 2} f_{i-1 / 2}\right]+\left[\alpha_{n+1 / 2} f_{n+1 / 2}-\alpha_{n-1 / 2} f_{n-1 / 2}\right]$, and $\bar{q}$ equal to the RHS of Eq. (10) so that it becomes

$$
f-\bar{q} \Delta t=f_{s}-\xi \Lambda \Delta t_{s} / \Delta \mu_{n} .
$$

It can also be seen from Eq. (10) that $\bar{q}$ can be separated into the sum of two terms $\bar{q}=\bar{q}^{v}+\bar{q}^{\mu}$, where

$$
\begin{aligned}
\bar{q}^{v} & =-\sum_{b} \frac{1}{\Delta v_{g}^{3}}\left[v_{g+1 / 2}^{2} J_{g+1 / 2}^{v}-v_{g-1 / 2}^{2} J_{g-1 / 2}^{v}\right], \\
\bar{q}^{\mu} & =-\sum_{b} \frac{1}{\Delta \mu_{n}}\left[J_{n+1 / 2}^{\mu}-J_{n-1 / 2}^{\mu}\right] .
\end{aligned}
$$

Equations (12) and (13), respectively, are the collision effects terms which change the magnitude of the velocity and the direction of test ions as they suffer small-angle scattering events.

If the term $\bar{q}^{v}$ is considered separately, it is found that by defining $B_{g}=$ $A \sum_{b} Z_{b}^{2} H_{b 2}(v) / A_{b}, C_{g}=\sum_{b} Z_{b}\left(H_{b 3}(v) / v+v^{2} H_{b 1}(v)\right) / 3$, and by using Eq. (4), it can be rewritten as

$$
\vec{q}^{v}=\frac{4 \pi \Gamma}{\Delta v_{g}^{3}}\left\{B_{g+1 / 2} f_{g+1 / 2}+C_{g+1 / 2} \frac{f_{g+1}-f_{g}}{\Delta v_{g+1 / 2}}-B_{g-1 / 2} f_{g-1 / 2}-C_{g-1 / 2} \frac{f_{g}-f_{g-1}}{\Delta v_{g-1 / 2}}\right\}
$$

Further, by using the interpolating relations of Chang and Cooper [13] which relate the velocity cell edged values of the distribution to the cell centered values, $\vec{q}^{v}$ can finally be written in the three-point difference form

$$
\begin{aligned}
\bar{q}^{v}= & \frac{4 \pi T}{\Delta v_{g}^{3}}\left\{f_{g-1}\left[\frac{C_{g-1 / 2}}{\Delta v_{g-1 / 2}}-B_{g-1 / 2} \delta_{g-1 / 2}\right]\right. \\
& +f_{g}\left[B_{g+1 / 2} \delta_{g+1 / 2}-\frac{C_{g+1 / 2}}{\Delta v_{g+1 / 2}}-\frac{C_{g-1 / 2}}{\Delta v_{g-1 / 2}}-B_{g-1 / 2}\left(1-\delta_{g-1 / 2}\right)\right] \\
& \left.+f_{g+1}\left[B_{g+1 / 2}\left(1-\delta_{g+1 / 2}\right)+\frac{C_{g+1 / 2}}{\Delta v_{g+1 / 2}}\right]\right\}
\end{aligned}
$$

where $\quad \delta_{g \pm 1 / 2}=\left\{1 / W_{g_{ \pm 1 / 2}}-1 /\left[\exp \left(W_{g \pm 1 / 2}\right)-1\right]\right\}, \quad$ and where $W_{g \pm 1 / 2}=$ 
$\Delta v_{g} B_{g \pm 1 / 2} / C_{g \pm 1 / 2}$. Similarly, by using Eq. (5), Eq. (13) can also be written in threepoint form as

$$
\begin{aligned}
\bar{q}^{u}= & \frac{4 \pi \Gamma}{\Delta \mu_{n}} \sum_{b} \frac{Z_{b}^{2}}{v^{3}}\left(H_{b 2}(v)-\frac{H_{b 3}(v)}{3 v^{2}}+\frac{2}{3} v H_{b 1}(v)\right) \\
& \times\left\{f_{n-1} \frac{\left(1-\mu_{n-1 / 2}^{2}\right)}{\Delta \mu_{n-1 / 2}}-f_{n}\left(\frac{\left(1-\mu_{n+1 / 2}^{2}\right)}{\Delta \mu_{n+1 / 2}}+\frac{\left(1-\mu_{n-1 / 2}^{2}\right)}{\Delta \mu_{n-1 / 2}}\right)\right. \\
& \left.+f_{n+1} \frac{\left(1-\mu_{n+1 / 2}^{2}\right)}{\Delta \mu_{n+1 / 2}}\right\} .
\end{aligned}
$$

\section{B. Solution of the Difference Approximation}

It can be seen from the difference approximation in the form of Eq. (11) that the terms which provide for streaming or spatial transport are on the RHS of Eq. (11) while the term $\bar{q} \wedge t$ which contains the collision effects is on the LHS. This suggests the possibility of using a splitting technique to solve the equation. If Eq. (11) is in fact split into two separate equations of the form

$$
\begin{aligned}
{\left[f-\bar{q} \Delta t_{s}\right]^{*} } & =\left[f_{s}-\frac{\xi \Lambda \Delta t_{s}}{\Delta \mu_{n}}\right]_{t=t_{s}}, \\
f & =f_{s}-\frac{\xi \Lambda \Delta t_{s}}{\Delta \mu_{n}}+\left[\bar{q} \Delta t_{s}\right]^{*}
\end{aligned}
$$

it becomes two fully implicit, consistently split equations, each of which contains the effects of spatial transport and collisions separately. The virtues of using consistent operator splitting for solving implicit systems are discussed in Ref. [14]. Equation (17) is an equation for $f^{*}$, an "intermediate" distribution, which modifies the test ion distribution for collision events while treating the streaming terms as known quantities evaluated at the old time $t_{\mathrm{s}}$ on the RHS. Equation (18) then uses this intermediate result as a known quantity through $\bar{q}^{*}=\bar{q}\left(f^{*}\right)$ and provides for the effects of spatial transport to yield the "updated" distribution $f$. In general, the value of the RHS of Eq. (17) at $t=0$ is given by an appropriate guess, but in our code $A$ was evaluated using the initial distribution function.

In order to solve Eq. (17), it is first noted that the factor $\bar{q}$ is the sum of the two three-point difference equations, Eq. (15) and Eq. (16). Since the RHS of Eq. (17) is a known quantity, Eq. (17) takes the form of a differenced Poisson equation [14] which can be written as

$$
E_{g}^{k} \psi_{n k}+G_{n}^{l} \psi_{l g}=S_{n g}, \quad k=g-1, g, g+1, \quad l=n-1, n, n+1,
$$

where the $E_{g}$ are the coefficients of the three-point group terms in Eq. (15), the $G_{n}$ are the coefficients of the three-point angle terms of Eq. (16), and $S_{n g}$ is the RHS of Eq. (17). The technique to be used for solving this equation is based on a method documented by Buzbee et al. [15]. A brief outline of this method will be given here. 
In general, $E$ and $G$ are not symmetric matrices but a matrix $D$ can be found which will symmetrize $E$ (or $G$ ) through the similarity transformation $\tilde{E}=D E D^{-1}$. I is easily shown that $D$ is diagonal in form so that the use of this transformation for $E$ in Eq. (19) yields

$$
\tilde{E} D \psi_{n}+G_{n}^{l} D \psi_{l}=D \mathbf{S}_{n},
$$

where the subscript appropriate to the group $g$ has been used as a vector index to simplify the notation. It is noted that the symmetric matrix $\tilde{E}$ has a complete set of eigenvectors given by $\tilde{E} \xi_{\alpha}=\lambda_{\alpha} \xi_{\alpha}$ so that the vectors $D \Psi$ and $D S$ can be expanded as

$$
\begin{aligned}
& D \Psi_{n}=\sum_{\alpha} a_{n \alpha} \xi_{\alpha}, \\
& D \mathbf{S}_{n}=\sum_{\alpha} b_{n \alpha} \xi_{\alpha}
\end{aligned}
$$

so that Eq. (20) can be rewritten as

$$
\left[G_{n}^{l}+\delta_{n}^{l} \lambda_{\alpha}\right] a_{l \alpha}=b_{n \alpha}
$$

where $\delta_{n}^{l}$ is the Kronecker delta. Equation (23) is recognized as a tridiagonal systern in $a_{l}$ for each $\alpha$. This system can be solved readily by a factorization of the tridiagonal matrix into upper and lower off-diagonal matrices. This is a standard technique [16] in matrix analysis, the details of which will not be given here. By then using Eq. (21), the solution $\psi_{l g}$ can be constructed as

$$
\psi_{l g}=\sum_{\alpha} a_{l \alpha} D_{g}^{-1} \xi_{\alpha g} .
$$

This is the intermediate distribution $f^{*}$. Note that the eigenvalues of $\tilde{E}$ need only be calculated once for the case where the background distributions remain isotropic since the coefficients in Eqs. (15) and (16) remain unchanged. But the construction [Eq. (24)] for the intermediate distribution must be performed at each time step since the RHS of Eq. (19) changes in time. These procedures are carried out for each zone in a given time step.

In order to complete the calculation for $\mathcal{f}$, Eq. (18) must be solved. Since the distribution $f^{*}$ is now known, $\bar{q}^{*}=\bar{q}\left(f^{*}\right)$ can be constructed. It is noted that Eq. (18) is identical to Eq. (10) except that the RHS of Eq. (10), i.e., $\vec{q}^{*}$, is now known. In considering Eq. (10), it can be seen that there are five quantities to be found at the updated time $t=t_{s+1}: f, f_{i+1 / 2}, f_{i-1 / 2}, f_{n-1 / 2}$, and $f_{n+1 / 2}$. In general, two of these, say, $f_{n-1 / 2}$ and $f_{i+1 / 2}$, are known from either boundary conditions or from a previous time step. The other three quantities can be related by some scheme so that a system of three quations in three unknowns can be formed.

We choose to use the diamond difference relations which linearly interpolate 
between quantities defined on a topologically rectangular region (Fig. 1) and are given by

$$
\begin{aligned}
& 2 f \simeq f_{n-1 / 2}+f_{n+1 / 2}, \\
& 2 f \simeq f_{i-1 / 2}+f_{i+1 / 2} .
\end{aligned}
$$

Using these equations to solve Eq. (10) for the distribution $f$ in terms of the known quantities yields the relation

$$
f=\frac{\left[\begin{array}{c}
\left\{\bar{q}^{*} \Delta t_{s}+f_{s} \frac{\Delta t_{s} \mu_{n}}{V_{i}} \frac{\Delta v_{g}^{4}}{\Delta v_{g}^{3}}\left[A_{i+1 / 2}+A_{i-1 / 2}\right] f_{i+1 / 2}\right. \\
\left.\left.+\frac{\Delta t_{s}}{V_{i}} \frac{\Delta v_{g}^{4}}{\Delta v_{g}^{3}} \frac{1}{\Delta \mu_{n}}\left[\alpha_{n+1 / 2}+\alpha_{n-1 / 2}\right] f_{n-1 / 2}\right\}\right]
\end{array}\right.}{\left\{1+\frac{\Delta t_{s}}{V_{i}} \frac{\Delta v_{g}^{4}}{\Delta v_{g}^{3}}\left[\frac{1}{\Delta \mu_{n}}\left(\alpha_{n+1 / 2}+\alpha_{n-1 / 2}\right)-\mu_{n}\left(A_{i+1 / 2}+A_{i-1 / 2}\right)\right]\right\}}
$$

where the alpha coefficients' recursion relation has been used to write the denominator in a more symmetric form. This equation can be used to solve for the updated distribution $f$ for all zones $i$, starting at the boundary of the sphere by calculating the cell centered distributions $f$ and then extrapolating inward for the cell edged distributions $f_{i-1 / 2}$. Since the calculation proceeds inward toward the center of the sphere, it should be performed only for angles directed inward to avoid the accumulation of numerical error [1], i.e., for the directions such that $-1 \leqslant \mu \leqslant 0$. A similar equation can be derived for outward directions by considering $f_{i-1 / 2}$ to be known and again using the diamond difference equations in conjunction with Eq. (10) to yield

$$
f=\frac{\left[\begin{array}{r}
\left\{\bar{q}^{*} \Delta t_{s}+f_{s}+\frac{\Delta t_{s} \mu_{n}}{V_{i}} \frac{\Delta v_{g}^{4}}{\Delta v_{g}^{3}}\left[A_{i+1 / 2}+A_{i-1 / 2}\right] f_{i-1 / 2}\right. \\
\left.+\frac{\Delta t_{s}}{V_{i}} \frac{\Delta v_{g}^{4}}{\Delta v_{g}^{3}} \frac{1}{\Delta \mu_{n}}\left[\alpha_{n+1 / 2}+\alpha_{n-1 / 2}\right] f_{n-1 / 2}\right\}
\end{array}\right]}{\left\{1+\frac{\Delta t}{V_{i}} \frac{\Delta v^{4}}{\Delta v^{3}}\left[\frac{1}{\Delta \mu_{n}}\left(\alpha_{n+1 / 2}+\alpha_{n-1 / 2}\right)+\mu_{n}\left(A_{i+1 / 2}+A_{i-1 / 2}\right)\right]\right\}} .
$$

The outward integrations can be started by using an isotropy condition at the center of the sphere which is just

$$
\begin{aligned}
f_{r=0, n_{\text {outward }}} & =f_{r=0, n_{\text {inward }},} \\
n_{\text {outward }} & =N N+1-n_{\text {inward }},
\end{aligned}
$$

where $N N$ is the maximum number of directions. This integration is done after all of the inward calculations have been performed. Once these streaming calculations are 


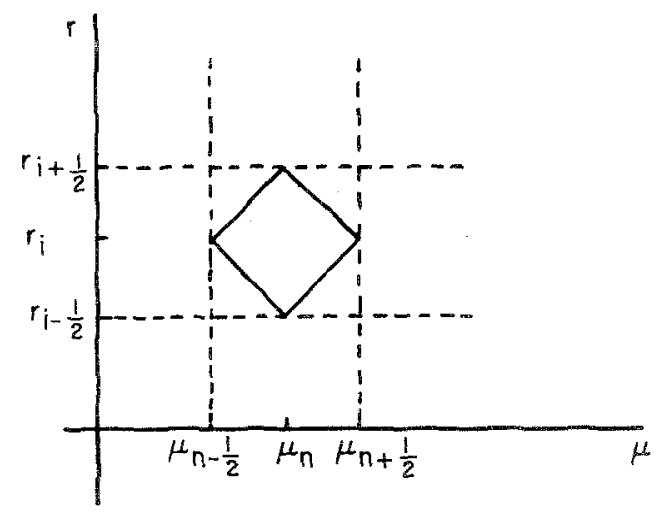

FIG. 1. The diamond structure of the interpolating procedure given by Eqs. (25) shown on a partial $r-\mu$ mesh.

complete, the solution is then iterated with Eq. (7) in order to obtain a consistent solution of the original fully implicit difference approximation, Eq. (10). In this way, $f(r, v, \mu, t)$ is calculated at the updated time $t=t_{s+1}$ for all zones, speeds, and angles.

\section{Results}

Of particular interest to researchers in inertial confinement fusion and in other related fields is the calculation of the amount of energy that fast ions deposit in a pellet plasma as they slow down due to collisional drag in the transport process. It is important to determine not only how this energy is distributed spatially, but also how it is partitioned to the background ions and electrons as well as how it depends on time. In this section, the results of calculations of this type, performed with the matrix factorization (MF) method, are presented.

The transport of test ions in a spherical D-T plasma is an example problem typical of benchmark problems which have evolved within the literature on CPT $[5-7,9,10]$. Here, the case of $3.5-\mathrm{MeV} \alpha$ particles being born isotropically in fusion events in the central zone of an otherwise nonreacting sphere of deuterium-tritium plasma is considered. The density of the background plasma is taken to be $0.2125 \times 10^{3} \mathrm{~kg} / \mathrm{m}^{3}$ at a temperature of $50 \mathrm{keV}$. It is chosen to compare the results of the MF calculations with those given by Mehlhorn and Duderstadt [7] since their method also allows for velocity space dispersion and since their treatment of streaming via discrete ordinates is similar. In order to match the zoning used in their modified neutronics code TIMEX-FP, 13 radial zones are used with the zone width taken to be $0.7742 \times 10^{-2} \mathrm{~m}$ while an 18-point speed mesh with four angles is used in velocity space.

In Figs. 2 and 3 , the fraction $E_{d} / E_{0}$ of the initial $\alpha$ particle energy $E_{0}$ deposited per zone to the background electrons and ions, respectively, is plotted for each zone. it 


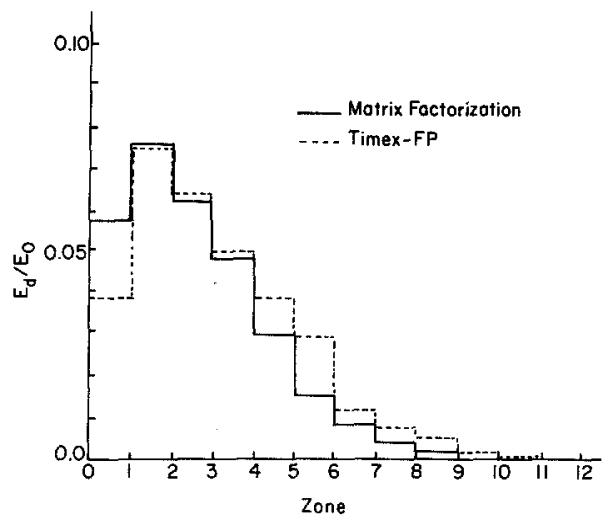

Fig. 2. Fraction of initial $\alpha$ particle energy $E_{0}$ deposited per zone to electrons for a source located in the central zone.

can be seen that the MF method yields results which are in good agreement with those reported in Ref. [7]. In both Figs. 2 and 3, the peaks of the spatial deposition profiles occur in the same zones and are nearly identical in magnitude. Similarly, the stopping lengths calculated by the MF method enjoy close agreement to those previously reported.

In order to study the effects of the dispersion in velocity space which the $\alpha$ particles undergo as they scatter on the plasma, the number of angles $N N$, used in the calculation was varied. In Figs. 4 and 5 the spatial deposition profiles are again given for electrons and ions separately. It is seen that by increasing the number of directions in which the $\alpha$ particle distribution function can be defined, for the case of deposition to the electrons, the spatial profile's peak is decreased while deposition to the outer zones is increased. In the case of the ions, the peak is also diminished but shifted to the right with the deposition to the outer zones again increasing. This

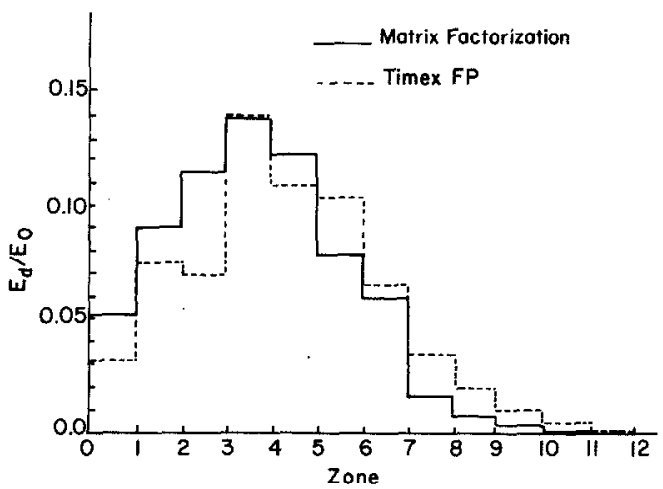

Fig. 3. Fraction of initial $\alpha$ particle energy $E_{0}$ deposited per zone to ions for a source located in the central zone. 


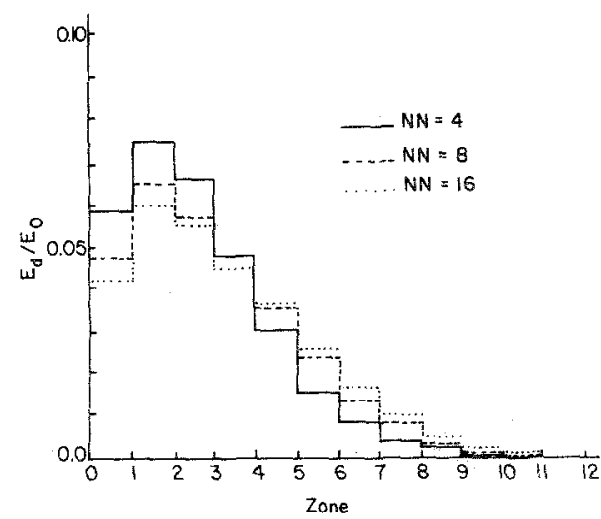

FIG, 4. Fractional deposition per zone to electrons using an increasing maximum number of angles $(N N)$.

behavior is to be expected for the following reasons. Since the initially isotropic $\alpha$ particles are at higher energies than the background electrons and ions, their distribution will depart from the isotropic form as they scatter in an attempt to reach a thermal equilibrium. Although the $\alpha$ energy may diminish after the first few collisions in zones near the center of the sphere, the energy is more directed in the outward direction in these zones. They will approach a thermal equilibrium after enough collisions have occurred along their path, so that their distribution will again acquire an isotropic character in the outer zones of the mesh. At this time the particles will have no prefered direction, so that the amount of backscattering will become the same as the amount of forward scattering, thus resulting in higher deposition to these outer zones. That this behavior is indeed the case is established by following the distribution of the cosine $(\mu)$ of the $\alpha$ particles' velocity vectors with respect to the radial vector as a function of time. In Fig. 6 this spectral information is shown for the center zone at $t=0$ while the curves at other times are appropriate to

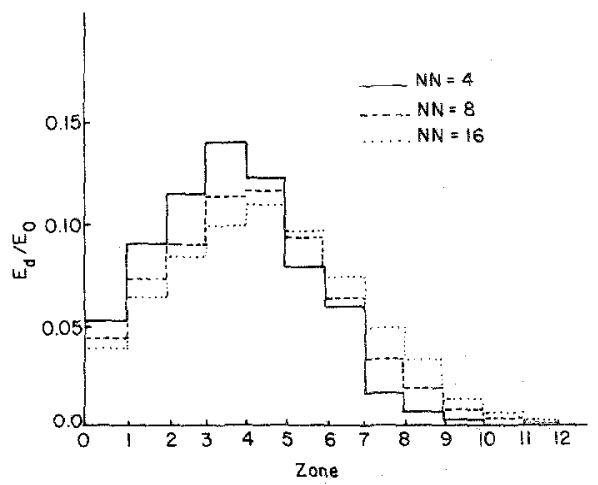

FIG. 5. Fractional deposition per zone to ions using an increasing maximum number of angles $(N N)$. 


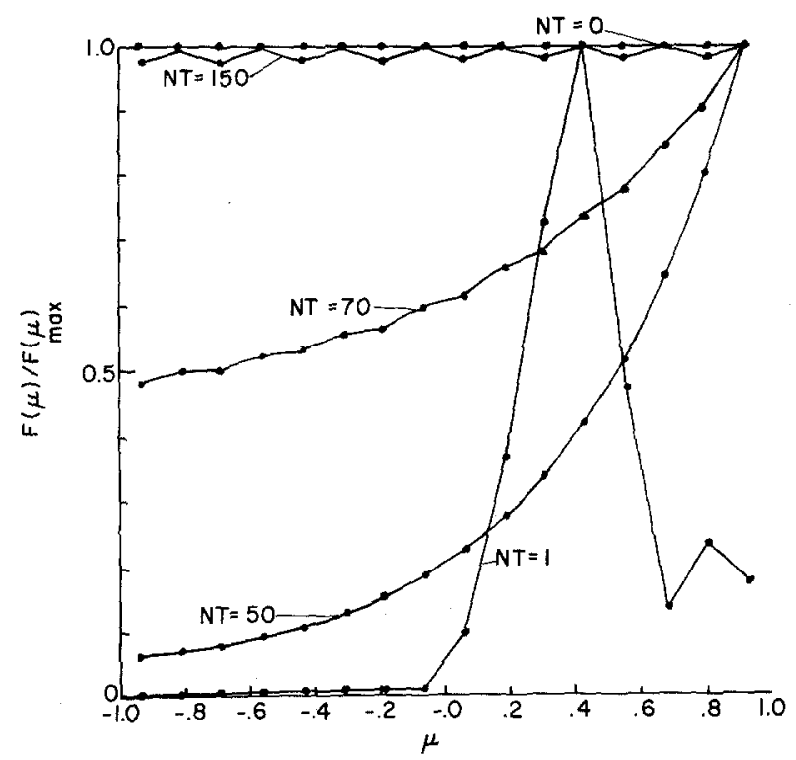

FIG. 6. Angular spectra of the distribution function $F$ (normalized), at the third position on the zone grid. At the first time iteration $(N T)$, the spectra is shown for the innermost zone.

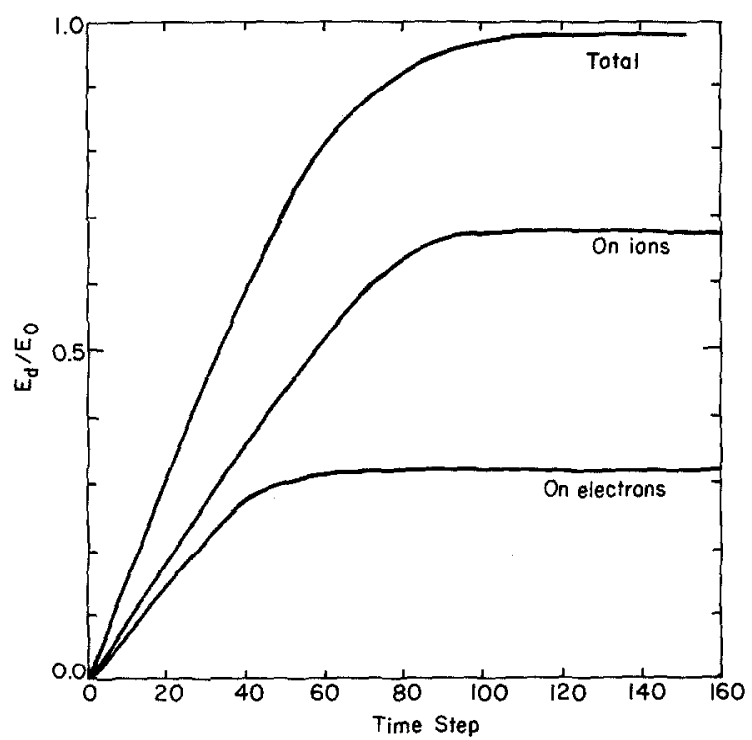

FIG. 7. Time dependent energy deposition history for deposition to both electrons and ions. 
the third zone on the mesh. It is seen that this distribution (normalized to unity on the abscissa) becomes peaked toward a positive cosine almost instantaneously showing that the $\alpha$ energy is highly directed toward the outer zones. At $N T=1$, in particular, the curve is seen to be discontinuous but clearly establishing a trend toward positive $\mu$. These discontinuities are due to the use of a large time step in the program. As time $(N T)$ progresses, the particles scatter and lose their energy and their distribution is seen to approach a Maxwellian at the background temperature. From this information, it can be concluded that by using too few angles in this type of calculation, the results may become biased in showing too much deposition in the first few zones and in ignoring the backscattering effects in the outer zones.

It is interesting to note that the plots in Fig. 6 contain data points which appear jagged. This is due to the use of a large time step in the algorithm, which gives rise to small fluctuations in the distribution information. Although this phenomenon could be detrimental in some algorithms, the MF method remained absolutely conservative and convergent.

In Fig. 7 the time dependent energy deposition history is shown for deposition both on electrons and on ions. As a check on the accuracy of this method, the curve showing the total energy fraction deposited to both ions and electrons was calculated using the appropriate moment of the LHS of the transport equation, Eq. (1). It can be seen that the code remained energy conserving.

It is noted that the total deposition fraction in time tends toward unity but becomes asymptotic at a value less than unity. This is, of course, due to the fact that the $\alpha$ particle does not lose all of its kinetic energy but only slows down to an energy defined by the temperature at thermal equilibrium.

The efficiency of the MF method is demonstrated in Figs. 8 and 9. The same computations described above for four angles, 13 zones, and 18 velocity grid points were performed using 150 time steps $(N T)$ at a time increment of 0.01 and then carried our again using 1500 time steps at $\Delta t_{s}=0.001$. Here the time increment $\Delta t_{s}$ is scaled to the slowing-down time of $\alpha$ particles due to electrons at $50 \mathrm{keV}$ which is

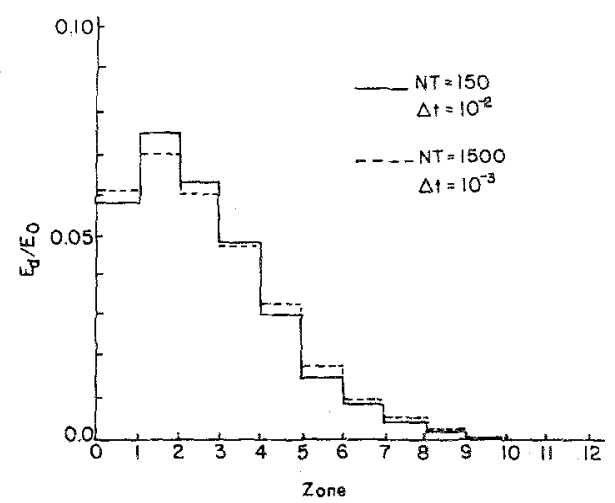

Fig. 8. Fractional deposition per zone to electrons for two time step sizes and the corresponding number of iterations $(N T)$. 


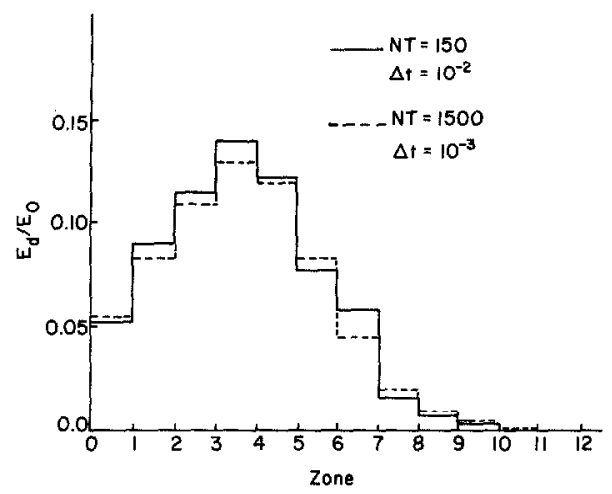

Fig. 9. Fractional deposition per zone to ions for two time step sizes and the corresponding number of iterations (NT).

equal to $8.47 \times 10^{-9} \mathrm{sec}$. It can be seen that very little accuracy is lost by using the larger time step. The calculation using 150 time steps required $5 \mathrm{sec}$. of CPU time on CRAY I computer.

\section{Conclusions}

It has been shown that the Fokker-Planck charged particle transport equation can be solved in a very efficient manner by splitting the difference approximation to yield two fully implicit equations, each of which is solved separately. One split equation contains the collision physics and modifies the distribution of test ions for these effects in its velocity variables $v$ and $\mu$, while the other equation corrects the distribution for spatial transport (streaming). A matrix factorization technique is used to solve the first of these equations (the "collision equation") while a standard forward extrapolation technique is employed to solve the "spatial" equation. Using the example of $3.5-\mathrm{MeV} \alpha$ particles, it was seen that the combination of these techniques in this algorithm yielded results which were in good agreement with those previously published. Furthermore, since the algorithm is fully implicit, it was also seen that large time steps could easily be used to generte accurate results in a minimum of CPU time.

Although the ditributions of ions and electrons describing the background plasma were postulated to be fixed Maxwellian distributions, the generalization to time, space, and velocity dependent distributions is straightforward within this algorithm. Further, the matrix factorization technique is not restricted to spherical geometry but can be applied in cylindrical and rectangular coordinates as well.

\section{ACKNOWLEDGMENTS}

The authors are grateful to Edward W. Larsen, Arthur A. Mirin, Thomas A. Mehihorn, and Foster Evans for helpful discussions. 


\section{REFERENCES}

1. G. I. BeLl AND S. Glasstone, "Nuclear Reactor Theory," Van Nostrand-Reinhold, New York, 1970.

2. J. J. Duderstadt AND W. R. MARTin, "Transport Theory," Wiley, New York, 1979.

3. J. Killeen AND K. D. MARX, "Methods of Computational Physics," Vol.9, Academic Press, New. York, 1969.

4. J. Killefn, A. A. Mirin, and M. E. Rensink, "Methods of Computational Physics," Vol. 16, Academic Press, New York, 1976.

5. P. A. Haldy and J. Ligou, Nucl. Fusion 17 (1977), 1225.

6. E. G. Corman, W. E. Loewe, G. E. Cooper, And A. M. Winslow, Nucl. Fusion 15 (1975), 377.

7. T. A. Mehlhorn and J. J. Duderstadt, J. Comput. Phys. 38 (1980), 86; T. A. Mehlhorn, Ph. D. dissertation, The University of Michigan, 1978.

8. J. E. MOREL, personal communication, Sandia Laboratories, 1979.

9. M. J. Antal and C. Lee, J. Comput. Phys. 20 (1976), 298; Nucl. Sci. Eng. 64 (1977), 379.

10. P. A. Haldy AND J. LigOU, Nucl. Sci. Eng. 74 (1980), 178.

11. L. Landau, Phys. Z. Sowjet. 10 (1936), 154.

12. M. N. Rosengluth, W. M. MacDonald, and D. L. Judd, Phys. Rev, 107 (1957), 1.

13. J. S. Chang and G. Cooper, J. Comput. Phys. 6 (1970), 1.

14. W. R. Briley and H. MCDonald, J. Comput. Phys. 34 (1980), 54.

15. B. L. Buzbee, G. H. Golub, And C. W. Nielson, SIAM J, Numer. Anal. 7, No. 4 (1970), 627.

16. R. D. Richtmyer, "Difference Methods for Initial-Value Problems," Interscience, New York, 1957. 